\title{
Gray Matter, White Matter and Cerebrospinal Volume in Schizophrenia and Subgroups: Mr Segmentation and Volumetric
}

\section{Study}

\begin{abstract}
Objective: Magnetic Resonance Imaging (MRI) has very high soft tissue resolution that's why we are able to discriminate gray matter and white matter y well voxel based volume analysis can reliably be made. In this study we aimed to investigate the volume differences of the total brain, gray matter, white matter and cerebrospinal fluid (CSF) between healthy controls and the subgroups of schizophrenia which are deficit syndrome (DS) and non-deficit syndrome (NDS).
\end{abstract}

\section{Introduction}

Schizophrenia is complicated phyciatric disease which may cause severe disability. Some of the schizophrenia patients may have only one period of psycosis. Some may have many psychotic periods and between the periods they may be relatively normal. People with chronic schizophrenia do not have normal periods and need a long term treatment. Chronic schizophrenia patients cannot live alone, they need be cared [1-3]. So schizophrenia is disease which may affect many people's lives.

Morphometry of the brain is popular subject in neuroscience. Magnetic Resonance Imaging (MRI) has very high soft tissue resolution so today we are able to discriminate gray and white matter very well and voxel based volume analysis can reliably be made [1]. MRI based morphometric studies are helping us for gaining data about the changes in the morphometry of the schizophrenia patients.

In this study we aimed to investigate the volume differences of total brain, gray matter, white matter and cerebrospinal fluid (CSF) between healthy controls and the subgroups of schizophrenia which are deficit syndrome (DS) and non-deficit syndrome (NDS).

\section{Materials and Methods}

Cases

Patients diagnosed with schizophrenia between the ages of 2270 monitored in the Department of Psychiatry at the Ege University Faculty of Medicine were included in the study. The diagnosis of schizophrenia was made according to DSM IV criteria. The patients were separated as DS and NDS with the use of Schedule for Deficit Syndrome in Schizophrenia. Those with axis 1 psychiatric disorder, those with medical disorders, and cases in the acute phase of schizophrenia (since the examination requires staying still for a long period and the patient compliance is low) were not included in the study. 30 schizophrenia patients (13 NDS, 17 DS) who fit the criteria

\section{Journal of}

Human Anatomy \& Physiology

\author{
Isil Yildiz ${ }^{1 *}$, Omer Kitis ${ }^{2}$ and Ali Saffet Gonul ${ }^{3}$ \\ ${ }^{\prime}$ Acibadem University Atakent Hospital, Istanbul, Turkey \\ ${ }^{2}$ Department of Radiology, Ege University, Izmir, Turkey \\ ${ }^{3}$ Department of Phsychiatry, Ege University, Izmir, Turkey
}

\section{*Address for Correspondence}

Isil Yildiz, Acibadem University Atakent Hospital, Istanbul, Turkey, E-mail: drisilyildiz@gmail.com

Submission: 22 March, 2018

Accepted: 13 July, 2018

Published: 18 July, 2018

Copyright: (c) 2018 Yildiz I, et al. This is an open access article distributed under the Creative Commons Attribution License, which permits unrestricted use, distribution, and reproduction in any medium, provided the original work is properly cited.

were examined with respect to prevalence of any organic pathology by routine (T1A, T2A, flair) cranial MRI examination. Volumetric analyses were carried out for 1 schizophrenia patient and 18 healthy control subjects, and a total of 30 subjects with kappa values over $0.98,14$ schizophrenia patients ( 7 of which were NDS) and 16 control subjects, were included in the study.

Control group was selected from healthy volunteers who were compatible with the patient group in terms of age, gender, and level of education. 18 people who did not have any psychiatric and medical disorders were included in the study. No organic pathology was detected in any of the routine cranial MRI examinations. When the kappa values were assessed in the volumetric analysis, two cases with kappa values below 0.98 were excluded from the study.

Details of the cranial MRI examination carried out for all of the cases for analysis are given below.

Study protocol was approved by the Board of Ethics in the Ege University Faculty of Medicine. Those who were included in the study were informed in detail about the study; and consent forms were obtained from the patients and patients' relatives.

MR imaging was carried out using a 1.5 Tesla device (Magnetom Vision to Symphony Upgrade, Erlangen, Germany) in the Department of Radio-Diagnostics at the Ege University Faculty of Medicine. Images were obtained from each subject in the patient and control groups in routine examination sequences along with the 3D FLASH, TSE T2, and PD sequences which are presented below in detail.

3D Flash: TR: $2300 \mathrm{msn}$, TE: $3.93 \mathrm{msn}$, Slice thickness: 1, Flip angle: 12, Dimension: 2D, FOV read: 256, FOV phase: 100, Bandwidth: 130, Echo spacing: 9.9

PD+T2 TSE: TR: 4500 msn, TE: 29 msn, Slice thickness: 1, Flip angle: 150, Dimension: 2D, FOV read: 256, FOV phase: 98 , Bandwidth: 130, Echo spacing: 14.3

Firstly, routine MRI examinations of the cases were evaluated with respect to prevalence of organic pathology. The cases with no organic pathology were included in the study. 
The images acquired in the sagittal plane were recorded in DICOM (Digital Image Communication) data format in order to carry out structural analysis. The images were exported to personal computers that had Linux operating system. All measurements were carried out by one person without any information about the names or diagnoses of the patients.

BRAINS2 software created by the Image Processing Lab at the University of Iowa was used for volumetric measurements. BRAINS2 is a versatile software package created for the purpose of the analysis and classification of human brain. It includes manual and automatic tools. It is frequently used for the analysis of MR images. The processing steps are:

1. Resampling/Aligning which consists of

a) Resampling.

b) Selection of Talairach parameters

c) Selection of cerebellum parameters.

2. Fitting

3. Tissue classification (segmentation) which consists of

a) Venous blood tracing

b) Classification

4. Neural network which consists of

a) Mask generation

b) ROI generation

c) Manuel adjustment (Trimming)

5. Volume measurement

\section{Resampling/Aligning}

a) Resampling (T1): The processes are carried out separately for T1-and T2-weighted images.

Resampling is carried out by the selection of two points specified in each axial, coronal, and sagittal planes in T1-and-T2-weighted series. In this process, interhemispheral fissure is realigned in the vertical plane and the line between anterior commissure (AC) and posterior commissure (PC) is realigned in the sagittal plane. Through these processes, the brain is relocated into a standard orientation. In this way, the differences based on locations are eliminated. However, anatomic differences still pose an obstacle towards full standardization.

\section{b) Selection of talairach parameters (T1)}

A coordinate system (grid) is created in the brain by the input of Talairach and cerebellum parameters. Through this grid, when the command is given to calculate the volume of a part of the brain, for example the hippocampus, the system makes calculations in the coordinates where the hippocampus is expected to be located. The processing steps are explained below.

\section{c) Selection of cerebellum parameters (T1)}

$4^{\text {th }}$ Ventricular Notch, post anterior and posterior is marked by locating the points where cerebellum disappears.
Resampling process is completed for T1A images. The data are recorded. Resampling process for T2A sections is carried out. The processing steps that are mentioned above process are performed identically for T2A sections, and the data are recorded.

Following the resampling process, the original images are transformed into a new 3 -dimensional image set with voxels of $1 \mathrm{x} 1 \mathrm{x} 1$ $\mathrm{mm}$. As a result, the images that will be processed are no longer in DICOM format but in the analyze format; and are standardized spatially as $\mathrm{x}, \mathrm{y}, \mathrm{z}, \mathrm{AC}-\mathrm{PC}$ midlines.

\section{Fitting}

Once the resampling process is completed for T1A and T2A series, it is necessary to render T1-and-T2-weighted images of which the data input was separate, anatomically compatible. For this, following the necessary modifications in the files, "FIT" command is given. The process is carried out automatically. It is necessary to check when the process is completed.

\section{Tissue classification}

The software has the information to distinguish between BOS, gray matter, and white matter during tissue classification; however, there is still a small amount of blood content in the acquired data. In order to distinguish blood, the blood areas in lateral dural sinuses which don't include any other tissue are selected manually; and thus the necessary data for the program to recognize and exclude these areas is obtained.

Classification process is carried out automatically. 8-bit images with signal intensities that can be classified are created. The expected intensities of images of which tissue classification is made are;

- $0=$ unexplained

- 1 = blood

- $10=$ pure BOS

- $70=50 \%$ BOS, $50 \%$ gray matter

- $130=$ pure gray matter

- $190=50 \%$ gray matter, $50 \%$ white matter

- $250=$ pure white matter

The program provides kappa values when it completes the classification process. These values should be above 0.98 . This means, for example, if the kappa value is 0.99 and an area is classified as white matter, the probability that this area is white matter is $99 \%$. If the kappa value is under 0.98 , the segmentation is considered to be failed, and the process should be performed from the start.

\section{Neural network}

\section{a. Mask generation}

Mask generation is carried out automatically after the command is given.

\section{b. ROI generation}

The purpose of this is to present the areas included in volume calculation in two dimensions for each section so that only the outer border of the mask remains linear. 
Citation: Yıldız I, Kitis O, Gonul AS. Gray Matter, White Matter and Cerebrospinal Volume in Schizophrenia and Subgroups: Mr Segmentation and Volumetric Study. J Hum Anat Physiol 2018;2(1): 5.

\section{c. Manuel adjustment}

The borders generated by the program are checked one by one for each section, and are manually adjusted in line with the points given below. Manual adjustment is carried out one by one for all coronal sections.

\section{Volume measurement}

This process is carried out automatically after the successful completion of the above steps. Once the necessary command is given, the whole brain, gray matter, white matter, and BOS volume are acquired in $\mathrm{cm}^{3}$.

\section{Statistical analysis}

MS-Excel and SPSS for Windows Version 15.0 (SPSS Inc., Chicago, IL, USA) package programs were used for statistical analyses. In all comparisons, $\mathrm{p}<0.05$ level was considered to be an indicator for statistical significance. Average values are presented as "arithmetic mean / standard deviation". Pearson's correlation test was used in the comparison of total brain, gray matter, white matter and BOS volumes with age and education level. ANOVA and post-hoc Bonferroni correction were used for the comparison of gray matter, white matter, BOS, and total brain volume in subjects with deficit and non-deficit schizophrenia and the control group.

\section{Results}

\section{Demographic characteristics}

14 patients (7 deficit and 7 non-deficit schizophrenia) who met the schizophrenia criteria according to DSM IV and 16 healthy control subjects compatible with the patient group in terms of age and gender were included in the study. 1 of the patients with deficit schizophrenia was female and 6 were male; while 3 of the patients with non-deficit schizophrenia were female and 3 were male. When the controls and the whole patient group are considered, there was no statistically significant difference between the groups with respect to gender ( $p>0.05)$. The evaluation was made using chi-square test and $\mathrm{p}<0.05$ was considered to be statistically significant.

Ages of schizophrenia patients varied between 22 and 70 (average 37.8 ), ages of patients with deficit schizophrenia varied between 22 and 40 (average 34.2), ages of patients with non-deficit schizophrenia varied between 24 and 70 (average 37.8), and the ages of the control subjects varied between 22 and 60 (average 34.0). Years of education was between 3-12 years in patients with non-deficit schizophrenia (average 8.9 years), 8-14 years in patients with deficit schizophrenia (average 7.5 years), and 5-18 years in the control group (average 15 years). ANOVA was used for comparison, and there was no statistically significant difference between the patient group and the control group with respect to age $(\mathrm{F}=2.38, \mathrm{df}=44, \mathrm{p}>0.05)$. There was a statistically significant difference between the patient group and the control group with respect to total years of education $(\mathrm{F}=15, \mathrm{df}=24$, $\mathrm{p}<0.05)$.

In order to compare patients with deficit and non-deficit schizophrenia with respect to duration of illness $\mathrm{T}$ test was used; and $\mathrm{p}<0.05$ was considered to be statistically significant. There was no statistically significant difference between the DS and NDS groups with respect to duration of illness ( $\mathrm{T}=2.01, \mathrm{p}>0.05)$.

\section{Gray Matter, White Matter, and BOS volumes in patient and control groups and their comparisons}

For the control group; gray matter volume varied between $706.62 \mathrm{~mm}^{3}$ and $918.63 \mathrm{~mm}^{3}$, while the average gray matter volume was $807.82 \mathrm{~mm}^{3}$; white matter volume varied between $338.93 \mathrm{~mm}^{3}$ and $572.46 \mathrm{~mm}^{3}$, while the average white matter volume was 427.53 $\mathrm{mm}^{3}$; BOS volume varied between $132.5 \mathrm{~mm}^{3}$ and $325.4 \mathrm{~mm}^{3}$, while the average BOS volume was $222.11 \mathrm{~mm}^{3}$; and the total brain volume varied between $1279.76 \mathrm{~mm}^{3}$ and $1675.03 \mathrm{~mm}^{3}$, while the average total volume was $1457.47 \mathrm{~mm}^{3}$. For patients with deficit schizophrenia; gray matter volume varied between $705.87 \mathrm{~mm}^{3}$ and $908.82 \mathrm{~mm}^{3}$, while the average gray matter volume was $792.16 \mathrm{~mm}^{3}$; white matter volume varied between $336.96 \mathrm{~mm}^{3}$ and $437.07 \mathrm{~mm}^{3}$, while the average white matter volume was $400.75 \mathrm{~mm}^{3}$; BOS volume varied between $140.15 \mathrm{~mm}^{3}$ and $355.79 \mathrm{~mm}^{3}$, while the average BOS volume was is $231.60 \mathrm{~mm}^{3}$; and the total brain volume varied between $1289.50 \mathrm{~mm}^{3}$ and $1661.04 \mathrm{~mm}^{3}$, while the average total volume was $1424.51 \mathrm{~mm}^{3}$. For patients with non-deficit schizophrenia; gray matter volume varied between $604.70 \mathrm{~mm}^{3}$ and $814.91 \mathrm{~mm}^{3}$, while average gray matter volume was $695.94 \mathrm{~mm}^{3}$; white matter volume varied between $279.85 \mathrm{~mm}^{3}$ and $525.70 \mathrm{~mm}^{3}$, while the average white matter volume was $391.59 \mathrm{~mm}^{3}$; BOS volume varied between 102.70 $\mathrm{mm}^{3}$ and $310.13 \mathrm{~mm}^{3}$, while the average BOS volume was $211.76 \mathrm{~mm}^{3}$; total brain volume varied between $1114.74 \mathrm{~mm}^{3}$ and $1535.20 \mathrm{~mm}^{3}$, while the average total brain volume was $1299.30 \mathrm{~mm}^{3}$. ANOVA was used for the comparison, and $\mathrm{p}<0.05$ was considered to be statistically significant. There was a difference between the groups with respect to gray matter and total brain volume $(\mathrm{p}<0.05)$.

The comparisons made using the post-hoc Bonferroni correction and with a $\mathrm{p}$ value of $<0.05$ were considered to be statistically significant. According to this, there was a statistically significant difference between patients with non-deficit schizophrenia and control subjects with respect to gray matter and total brain volume $(\mathrm{p}<0.05)$.

Relationship between gray matter, white matter, bos, total brain volume and age in patients with deficit schizophrenia

In the evaluation carried out with Pearson Correlation Analysis, $\mathrm{p}<0.05$ was considered to be statistically significant. While there was no significant relationship between age and gray matter, BOS volume, and TBV in patients with deficit schizophrenia $(\mathrm{p}>0.05)$; there was a statistically significant relationship between white matter and age $(\mathrm{p}<0.05)$.

\section{Discussion}

There is a rapid growth of head and brain size in the first 2 years of life. In a study, Reiss et al. found that there were very small changes in the brain size after age 5 [4]. Other studies showed that nutrition, prematurity, and birth complications might be risk factors for small brain or small head size [5]. Environmental factors, normal development or both stabilize brain development in the early years of life. Intracranial volume remains as a basic parameter for the rest of life. DBV can be used as an indicator of the optimal brain volume in maturity or before the onset of illness. TBV can be calculated by using MRI. TBV and TBV differences in neuropsychiatric diseases are related to premature affective disorders, developmental disorders, childhood-onset psychotic disorders, and schizophrenia. 
The neurodevelopmental theory widely accepted for schizophrenia pathogenesis suggests that schizophrenia is a result of a simple and structural deficit regarding the early developmental phase in the early periods of life. The time between a lesion that has been formed at the early period and the onset of a disease is explained by the genetic changes in the neurodevelopmental mechanisms such as cortical pruning which occur in puberty. Gray matter is responsible for the growth in the human brain, rather than the white matter. Therefore, according to this hypothesis, the areas where we expect to find differences in schizophrenia patients in radiological studies are gray matter and TBV (since no change is expected in the white matter). In our study, a significant difference was found between NDS and control groups with respect to TBV and gray matter volume. TBV and gray matter volume was lower in NDS patients. This result we obtained is supportive of the neurodevelopmental hypothesis. Similar results were obtained in other studies as well. In a study conducted by Zipursky et al. on 22 male and 20 healthy controls aged 23-45, it was found that there was a statistically significant decrease in the gray matter volume in schizophrenia patients compared to the control group [6]. In a meta-analysis, Steen et al. examined 52 cross sectional studies that included 1454 patients and 16 longitudinal studies that included 465 patients; and concluded that TBV decreased in schizophrenia patients compared to healthy controls [7]. Sullivan et al. in their study which included 128 schizophrenia patients, found a decrease in the gray matter volume in schizophrenia patients [8].

Factors such as age, years of education, and duration of illness which may have an effect on gray matter volume and total brain volume were taken into consideration during the evaluation.

When each group (DS, NDS, control) was evaluated within itself, contrary to expectations, no relationship was found between age and gray matter and total brain volume. The reason for this may be the small number of patients. There are many studies in the literature showing that the total brain and gray matter volume decreases with age. In a study conducted on 176 male and 205 female patients aged between 21 and 79, it was found that atrophy was related to age especially after the age of 40 [9]. In another study, 130 healthy subjects were evaluated via cranial CT, and the findings demonstrated atrophy associated with age, especially in subjects over 40 [10]. In a volumetric study conducted with 69 healthy people between the ages of 18-80 via MR, a negative correlation was found between the total brain volume and age [11].

In our study, since there was no statistically significant difference between the groups with respect to age, age ceased to be a factor for volume comparison.

There were no studies in the literature review that analyzes the effect of education on total brain and gray matter volumes in schizophrenia patients. However, in a study carried out with 320 people aged between 66 and 90, BOS volume was considered to be a primary indicator of brain atrophy; and it was shown that every additional year in education was related to $1.77 \mathrm{ml}$ decrease in BOS volume. Thus, studies based on years of education can be carried out via $\mathrm{CT}$ while some can be carried out with MR and volumetric analyses can be carried out using different methods. However, it is possible to state that in most of the studies carried out using current methods, contrary to expectations, structural abnormalities are encountered more in the NDS group compared to the DS group.
As in other current studies, we used ESC in our study. The method we used for volume calculation is highly reliable and allows one to use three sequences (T1, T2, and PD) unlike other morphometric methods; and therefore can distinguish between anatomic structures especially during manual adjustment, more reliably. In the segmentation process, whether a tissue is gray matter, white matter, or BOS is evaluated with $99 \%$ accuracy. This is shown by the obtained kappa values. If the kappa values are below $98 \%$, in other words, the accuracy of tissue classification is below $98 \%$, the processes is repeated; which in turn significantly increases reliability.

The similar results found in this study and other current studies allow us to question the idea that DS is a severe form of schizophrenia $[12,13]$, and think about the different processes regarding the etiology and pathogenesis between subgroups. There are significant reflections of this. The studies based on the assumption that schizophrenia is a core disease per se both presented weak findings, and the findings of these studies were not verified in the confirmation studies. There is a serious heterogeneity between the patients diagnosed with schizophrenia. Causal and neuropathological findings that are valid for some patients are not found in others. The characteristics of the disease show clear differences between the patients; and the symptomatic components of the disease demonstrate weak relations with each other for each individual. In other words, schizophrenia is not a homogenous disease, and presents itself through different pathological fields which have weak relationships with one another. Therefore, schizophrenia should be considered more as a syndrome.

Radiology and volumetric studies have had important contributions in the research of the etiology and pathogenesis of schizophrenia. Brain morphometry has emerged as one of the most dynamic fields in clinical neuroscience. In the recent years, with the development of new measurement techniques and development of image resolution, surprising results have been found which are similar to those in our study.

The "deficit syndrome" which is a unique type of schizophrenia results in the loss of valuable human experience and negatively effects long term functional outcomes. Further research focused on the causes and treatment of this condition is required. We believe that morphometric studies conducted on specific regions of the brain regions may be helpful.

In future research on schizophrenia, the focus of the therapeutic studies will not consider schizophrenia as a core disease; rather specific fields of pathology will be analyzed which in turn will open the door for target aimed medication treatments. In order to understand the genetic causality, genetic studies will substitute the genetics of schizophrenia as a syndrome for the genetics of pathological fields. As a result, the fields of interest in the medication for schizophrenia will shift from producing dopamine antagonists to new molecular targets and therapeutic profiles. It is expected that especially MR spectroscopy and PET will contribute to research in this field.

\section{References}

1. Ceylan E, Cetin M (2005) Sizofreni-I. Istanbul pp.727-797.

2. Kaplan HI, Sadock BJ (1985) Psyciatric report. Lippincott Williams \& Wilkins, Philadelphia, USA, pp. 1213-1238.

3. Karno M, Norquist GS (1985) Schizophrenia: epidemiology. Lippincott Williams \& Wilkins, Philadelphia, USA, pp. 986-1012. 
Citation: YIldız I, Kitis O, Gonul AS. Gray Matter, White Matter and Cerebrospinal Volume in Schizophrenia and Subgroups: Mr Segmentation and Volumetric Study. J Hum Anat Physiol 2018;2(1): 5.

4. Kwon JS, Shenton ME, Hirayasu Y, Salisbury DF, Fischer IA, et al. (1998) MRI study of cavum septi pellucidi in schizophrenia, affective disorder, and schizotypal personality disorder. Am J Psychiatry 155: 509-515.

5. Courchesne E, Chisum HJ, Townsend J, Cowles A, Covington J, et al. (2000) Normal brain development and aging: quantitative analysis at in vivo MR imaging in healthy volunteers. Radiology 216: 672-682.

6. Zipursky RB, Lim KO, Sullivan EV, Brown BW, Pfefferbaum A (1992) Widespread cerebral gray matter volume deficits in schizophrenia. Arch Gen Psychiatry 49: 195-205.

7. Steen RG, Mull C, McClure R, Hamer RM, Lieberman JA (2006) Brain volume in first episode schizophrenia: systemic review and meta-analysis of magnetic resonance imaging studies. Br J Psyciatry 188: 510-518.

8. Sullivan EV, Lim KO, Mathalon D, Marsh L, Beal DM, et al. (1998) A profile of cortical gray matter volume deficits characteristic of schizophrenia. Cereb Cortex 8: 117-124.
9. Takeda S, Matsuzawa T (1985) Age related brain atrophy: a study with computed tomography. J Gerontol 40: 159-163.

10. Ito M, Hatazawa J, Yamaura H, Matsuzawa T (1981) Age related brain atrophy and mental deterioration--a study with computed tomography. $\mathrm{Br} \mathrm{J}$ Radiol 54: 384-390.

11. Gur RE, Mozley PD, Resnick SM, Gottlieb GL, Kohn M, et al. (1991) Gender differences in age effect on brain atrophy measured by magnetic resonance imaging. Proc Natl Acad Sci U S A 88: 2845-2849.

12. Gur RE, Mozley PD, Shatasel DL, Cannon TD, Gallacher F, et al. (1994) Clinical subtypes of schizophrenia: differences in brain and CSF volume. Am J Psyciatry 151: 343-350.

13. Sigmundsson $T$, suckling $\mathrm{J}$, Maier $\mathrm{M}$, Williams $\mathrm{S}$, Bullmore $\mathrm{E}$, et al. (2001) Structural abnormalities in frontal, temporal and limbic regions and interconnecting white matter tracts in schizophrenic patients with prominent negative symptoms. Am J Psyciatry 158: 234-243. 